\title{
Crafting Compelling Personal Statements
}

\author{
Latha Chandran $^{1}$ (D) $\cdot$ Arathy S. Chandran ${ }^{2}$. Janet E. Fischel ${ }^{2}$
}

Received: 20 February 2020 / Accepted: 7 October 2020 / Published online: 4 November 2020

(C) Academic Psychiatry 2020

A personal statement is a professional essay about the writer, trying to persuade the reader to positively consider one's qualifications, key traits, experiences, and aspirations. The goal of the personal statement is to inform the reader about the writer in a way that promotes the writer for selection or further consideration. Our combined experience with reviewing personal statements for admissions, awards, and promotion committees is seven decades long. This article discusses the writing and use of personal statements in the context of both that experience and information in the literature. We focus predominantly on guidance for personal statements prepared by trainees for entrance to their next step, such as acceptance to medical school or graduate medical education. However, even those preparing materials for a competitive award or faculty promotion might find the information relevant. Particularly in psychiatry, but important to any health sciences field, the ability to communicate effectively and demonstrate the highest standards of professionalism in attitudes and behaviors is paramount; the personal statement provides a forum to display those characteristics. If one is seeking admission to medical school, welldescribed experiences that effectively communicate the reasons to do so would be valuable to include. If applying for residency or fellowship, the goal may shift toward affirmation of one's motivation, experiences, and skills to pursue this particular field. All this, without simple factual repetition of one's curriculum vitae $(\mathrm{CV})$, and without exaggeration or arrogance.

An excellent personal statement for medical school entrance, in our experience, was one starting with a brief scenario drawn from working in health care in a developing country; another identified the characteristics of an ancient scholar and physician, elaborating on the qualities the writer emulated, and clarifying why he chose further studies to gain similar

Latha Chandran

lchandran@med.miami.edu

1 University of Miami, Miami, FL, USA

2 Renaissance School of Medicine at Stony Brook University, Stony Brook, NY, USA skills. A third described a particularly devastating illness in a family member. These were impressive when coupled with insight into the writer's motivation to pursue a health professions career. The reader could glean the writer's observational, analytical, and reflective skills. A brief scenario such as these need not launch the essay, but is a popular approach.

What differentiates a memorable personal statement from the rest is that the writer is genuine, the information is clear and interesting, the writing is disciplined, and the writer is able to thread the theme of an opening vignette or experience further than simply reporting on it. Thus, the rest of the statement and a review of the accompanying $\mathrm{CV}$ each show some accomplishments or next choices in education or activities supporting the claim that the experience described was seminal in pursuing this next step in the writer's career. In contrast, stories of single patient care experiences with minimal connection to other parts of the narrative often feel inauthentic in their impact on the author's career journey.

In the context of an interesting discipline known as genre analysis, experts in English pedagogy have looked at a variety of genres to glean patterns in the narrative and expectations about content. In that regard, Ding points out two interesting challenges about personal statements in academic medicine: first, the writer is not yet part of that culture or community, and so is unfamiliar with the expectations of how the essay will be judged; secondly, the personal statement provides "allowance for creativity and individuality ... space for narratives and stories, and its goal [is] both to inform and to persuade." [1] Thus, we should not underestimate the value of both mentorship and guidance from informed sources for crafting a compelling personal statement.

What are the dos and don'ts of writing personal statements in academic medicine? How does one craft a compelling personal statement? In truth, there is no singular right approach to the personal statement, and success cannot be assured, because the personal statement is typically one of several sources of information provided to reviewers. The National Residency Matching Program's 2018 survey of residency program directors across all specialties showed that $78 \%$ of 1233 programs cited the personal statement as a consideration for selection for 
interview, and $56 \%$ of 1208 programs included it in ranking consideration [2]. In psychiatry, those figures are $90 \%$ of 58 programs for interview selection and $74 \%$ of 58 programs for ranking decisions [2]. The internet provides information from consulting agencies, numerous tips, topics to write about, samples, and possible "hooks" to gain reader attention. There are websites that offer ghostwriting of personal statements for purchase [3]. Such resources are not unique to academic medicine or guided by research evidence. In fact, there is limited literature with supporting research on best practices surrounding the writing of personal statements in academic medicine. Nonetheless, what is currently available allows us to glean some helpful findings.

A prospective study of an entering class of 176 medical students in the UK found that the information in the personal statements (in a detailed coding scheme for such things as motivation and hobbies) as well as "conscientiousness" in the personality assessments of the students predicted clinical aspects of their training while the letters of references from teachers had no predictive value [4]. In applications to psychiatry residency programs, advice includes using the personal statement to "bring to light information about the applicant not found elsewhere in the application." [5] Other advices include information on how and why the applicant selected the field, brief explanation of academic difficulty, if any, and what was learned from it, and perhaps information on future plans [5]. Furthermore, 131 US program directors in anesthesiology were surveyed about how they used personal statements; with 70 respondents, although only $41 \%$ considered it very or somewhat important for selecting interview candidates, and the vast majority "always" or "sometimes" used the essay for interview selection and during actual interviews [6]. Those same researchers evaluated ratings by four reviewers of 670 de-identified personal statements in applications to their institution's anesthesiology program. Quality of the essays was rated on a 5-point scale from poor to superior for eight characteristics, such as grammar, compelling, and original/personal. Graduation from a US or Canadian medical school, female gender, and younger age were associated with what the reviewers rated as "higher quality" personal statements [6]. In a study of over 2000 internal medicine residency applicants' personal statements, female applicants focused more on team membership and emotional aspects of doctoring, while male applicants wrote more frequently about personal qualities and skills [7].

Although a different graduate education pathway, virtually all doctoral programs in psychology and counseling require the submission of a personal statement. It is consistently rated as the most important document when selecting doctoral students [8]. In these situations, discussion of the candidate's research interests and understanding of the field were considered paramount in being selected [8]. With 97 respondents from the 367 polled (26\% response rate), factors viewed positively included discussion of one's strengths and eagerness to learn more in the field as well as better alignment between one's interest and the program goals, while factors such as unprofessional tone, exaggerated altruism, poor writing, and lack of awareness of the program's goals were deemed damaging [8]. In a 2006 study polling the chairs of graduate admissions committees in psychology, four areas of concern emerged as damaging: excessive altruism, excessive self-disclosure, being professionally inappropriate, and a focus on personal mental health. While interesting, the work should be interpreted with caution, as just $19 \%$ of surveys were returned. Furthermore, in the category of personal mental health, reviewers gave examples of statements showing "emotional instability" that precluded consideration for selection [9].

The disclosure of mental health-related issues in personal statements is a complex consideration. As reported by Pheister et al., survey results of residency program directors indicate that applicants with a disclosure of depression were at higher odds of not receiving an interview invitation and ranking for match than those who had no such disclosure [10]. However, even with a disclosed illness, a strong applicant would fare better than an average applicant without such disclosure. The authors conclude that disclosure of mental illness puts the applicant at a disadvantage that could be overcome by the strength of the rest of the application [10]. A study of medical students from the University of New Mexico (51\% response rate for 349 students) indicated that the majority of students who had a mental health diagnosis would not disclose it for a board license application or for a residency application through ERAS [11]. The most common reason cited for such reluctance was the fear of being stigmatized and of not matching. In sum, this is a personal decision that deserves careful and deliberate thought. Applicants should be aware that confidentiality of information is not assured, and applications are often reviewed by multiple committee members. From another perspective, one can argue that experience with an illness might enhance insight and compassion. Based on the available research evidence and on our collective experience, we would recommend such disclosure only when the applicant has (a) an excellent reason to offer it, (b) managed the difficulties effectively, and (c) demonstrated skills to continue training successfully.

\section{When Does One Use a Personal Statement?}

Personal statements are used for diverse purposes. The important point here is that, if a personal statement is requested, it is to your benefit to provide one. It is an opportunity to present information about oneself that provides the reader a rich context for understanding your preparation and competitiveness for selection from a pool of very likely well qualified applicants. A quick aside here. We find it fairly easy to identify the occasional personal statement that was probably "recycled" for residency or fellowship applications after it was initially 
prepared for college or medical school applications. Why? The cut-and-paste substitutions typically do not demonstrate the intimate connection to the current purpose.

The submission of your CV does not preclude the importance of a personal statement. These two documents serve different functions. The CV is usually structured for the detailed facts of your academic life in each domain of your work. It is provided in a format that is often highly organized by headings (e.g., education, licensures, committee work) but without page limit. The personal statement is preferably $1-2$ single-spaced pages, and invites you to illuminate aspects of your education or experience. While organized, the narrative is not structured by a pre-existing format. Thus, you can be subjective or objective, in places, about the impact of your activities and possible future plans.

\section{Questions to Consider When Writing Your Personal Statement}

Consider the following questions as you begin to craft your personal statement.

1. Why do you want to join this field or educational program? Why now?

2. What is noteworthy about you, your work or life experiences, and how do those experiences link to this next career step?

3. What characteristics and experiences qualify you for this?

4. What are your future goals after completing this training?

Keeping those questions in mind, the personal statement should demonstrate evidence of self-reflection and learning through experience. As a succinct representation of your personal qualities, your journey so far, your accomplishments, lessons learned and your anticipated path forward, the essay might include your vision for yourself professionally as well as traits such as perseverance, integrity, commitment, or enthusiasm. Consider indicating how your vision for yourself aligns with the institution's or program's vision. A compelling personal statement often has evidence of reflection on your career path thus far and how your thinking has evolved along that journey. Evidence of a growth mindset and brief, careful self-analysis helps the reader learn about your strengths. Carefully select specific examples to illustrate your qualities as highly desirable for the purpose of the application.

\section{How Does One Organize a Personal Statement?}

Before starting to write your personal statement, be clear about its intended purpose. What is it for? Who will read it?
Why do you merit selection? This will help hone what you want to include. Thus, the first step is to start with both the reader and your goal in mind. Which activities have the highest potential to influence the reader to favor selecting you? Remain truthful, without exaggeration.

We suggest three sections to the personal statement: an introduction, a body, and a conclusion. The introduction should engage the reader. The body of the personal statement typically has 3-4 paragraphs in a logical order. For example, if it is created for a residency application, these paragraphs might describe experiences that influenced your selection of this field, or address research you have done that captured your interest in this field. Emphasize your personal skills that may be highly valued in the field, thereby distinguishing yourself from others. The conclusion should be a synthesis of your successes, a reflection on your career so far, perhaps key lessons learned along the way. This is where you would likely demonstrate your vision, sense of purpose, and alignment with priorities the reader is seeking. A compelling personal statement leaves the reader inspired about you.

We consider it ideal to write your personal statement in the first person and in an active voice. Having the draft of your personal statement reviewed by trusted colleagues and advisors is strongly recommended as they can be extremely helpful in identifying gaps or areas of ambiguity.

\section{Common Pitfalls to Avoid}

Personal statements and application materials can be dishonest in several ways. We felt it was important to include a discussion of plagiarism, ghost writers, purchasing an essay, or including false information. Each is a highly unethical practice. Feller reviews an array of deceptions in studies of medical student, residency and fellowship applications, with false claims of honorary society memberships, manuscript authorship or acceptance, roles in extracurricular activities, falsified official documents, and plagiarism in the personal statement [3]. He points out that inadequate training about plagiarism or inadvertent deceit might be in play, but the frequency of occurrence is sobering, and speaks to the importance of mentorship and guidance. Using other people's work without appropriate attribution is academic dishonesty and is extremely unprofessional.

It is prudent to avoid lengthy descriptions of failures, and if failures are important to the story, avoid externalizing blame. Additionally, vignettes that include patients or their family members must remove any specific identifying information. In a personal statement draft, a trainee had written that general pediatrics would be monotonous, with mostly managing middle ear infections. Imagine if the reader's expertise included career-long research involving childhood ear infections! Sarcasm is not recommended. Humor can be risky in written narrative. If used, it needs to be carefully presented. As an example, a well-constructed 
essay, organized in the structure of a SOAP note with brief expressions of humor, was well-received, in our experience.

\section{Summary}

Preparing a compelling personal statement can seem like a daunting task. While it deserves time, thought, and careful attention, it might best be approached as an opportunity to showcase yourself. Armed with information about how to select what you wish to share, ways to illustrate your unique and genuine competencies, how to organize the essay, and what the reader might be seeking from your essay, the preparation of a clear and compelling personal statement can be well within your grasp.

Acknowledgments The authors wish to gratefully acknowledge the support provided by Dr. Jeff Swain.

\section{Compliance with Ethical Standards}

Disclosures On behalf of all authors, the corresponding author states that there is no conflict of interest.

\section{References}

1. Ding H. Genre analysis of personal statements: analysis of moves in application essays to medical and dental schools. Engl Specif Purp. 2007;26(3):368-92.
2. National Resident Matching Program, Data Release and Research Committee. Results of the 2018 NRMP Program Director Survey. National Resident Matching Program, Washington, D.C. 2018.

3. Feller E. Deceit, lies and plagiarism in residency applications. R I Med J (2013). 2019;102(2):8-9.

4. Fergeson E, James D, O'Hehir F, Sanders A. Pilot study of the roles of personality, references, and personal statements in relation to performance over the five years of a medical degree. BMJ. 2003;326(7386):429-31.

5. Bak MK, Louie AK, Tong LD, Coverdale J, Roberts LW. Down to earth column: applying to psychiatry residency programs. Acad Psychiatry. 2006;30(3):239-47.

6. Max BA, Gelfand B, Brooks MR, Beckerly R, Segal S. Have personal statements become impersonal? An evaluation of personal statements in anesthesiology residency applications. J Clin Anesth. 2010;22(5):346-51.

7. Osman NY, Schonhardt-Bailey C, Walling JL, Katz JT, Alexander EK. Textual analysis of internal medicine residency personal statements: themes and gender differences. Med Educ. 2015;49(1):93102.

8. Davis KM, Doll JF, Sterner WR. The importance of personal statements in counselor education and psychology doctoral program applications. Teach Psychol. 2018;45(3):256-63.

9. Appleby DC, Appleby KM. Kisses of death in the graduate school application process. Teach Psychol. 2006;33(1):19-24.

10. Pheister M, Peters RM, Wrzosek MI. The impact of mental illness disclosure in applying for residency. Acad Psychiatry. 2020;44: 554-61. https://doi.org/10.1007/s40596-020-01227-8.

11. Fletcher I, Castle M, Scarpa A, Myers O, Lawrence E. An exploration of medical student attitudes towards disclosure of mental illness. Med Educ Online. 2020;25:1. https://doi.org/10.1080/ 10872981.2020.1727713.

Publisher's Note Springer Nature remains neutral with regard to jurisdictional claims in published maps and institutional affiliations. 